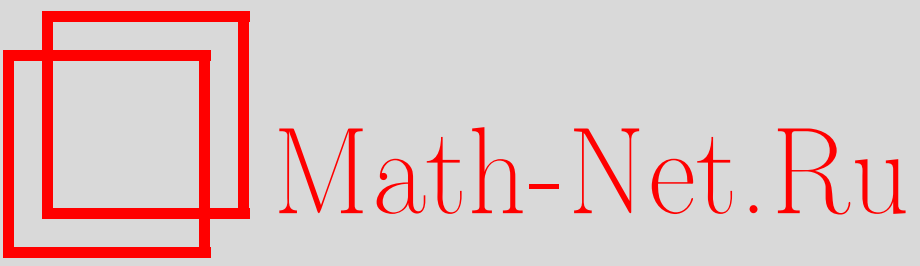

Ю. С. Вернов, М. Н. Мнацаканова, Высокоэнергетические соотношения между амплитудами упругого рассеяния частицы и античастицы, ТМФ, 2002, том 132, номер 2, 198-210

DOI: https://doi.org/10.4213/tmf356

Использование Общероссийского математического портала Math-Net.Ru подразумевает, что вы прочитали и согласны с пользовательским соглашением

http: //www . mathnet.ru/rus/agreement

Параметры загрузки:

IP : 35.173 .137 .237

26 апреля 2023 г., 16:33:48 
ТЕОРЕТИЧЕСКАЯ

И МАТЕМАТИЧЕСКАЯ

ФИЗИКА

Том 132, № 2

август, 2002

(C) 2002 г. $\quad$ Ю.С. Вернов* ${ }^{*}$ М. Н. Мнацаканова ${ }^{\dagger}$

\section{ВЫСОКОЭНЕРГЕТИЧЕСКИЕ СООТНОШЕНИЯ МЕЖДУ АМПЛИТУДАМИ УПРУГОГО РАССЕЯНИЯ ЧАСТИЦЫ И АНТИЧАСТИЦЫ}

Найдены условия, при которых отношение полных сечений рассеяния частицы и античастицы $\sigma_{+}(s) / \sigma_{-}(s) \rightarrow 1$ при $s \rightarrow \infty$. Если амплитуды упругого рассеяния вперед асимптотически становятся чисто вещественными, их отношение стремится $\mathrm{K}-1$. Доказано, что $\sigma_{+}(s) / \sigma_{-}(s) \cong 1$, если $\sigma_{+}(s) \gg \pi / m_{\pi}^{2}, m_{\pi}$ - масса $\pi$-мезона. Показано, что для ряда процессов полученные асимптотические соотношения имеют конечноэнергетические аналоги.

Ключевые слова: аксиоматическая теория поля, высокоэнергетическое рассеяние, аналитичность, унитарность, перекрестная симметрия, теорема Померанчука.

\section{1. ВВЕДЕНИЕ}

Для нас большая честь представить статью для этого номера журнала ТМФ, посвященного юбилею Анатолия Алексеевича Логунова, труды которого сыграли выдающуюся роль в создании современной теории элементарных частиц.

Одним из важнейших достижений теории элементарных частиш является доказательство аналитичности амплитуд рассеяния в $s$ - и $t$-плоскостях [1]-[5] ( $s, t$ и $u$ - стандартные инвариантные переменные). Условия аналитичности, унитарности и перекрестной симметрии накладывают ряд принципиальных ограничений на поведение амплитуд рассеяния, особенно в области высоких энергий.

Одно из таких ограничений было найдено Померанчуком [6] и получило название теоремы Померанчука. Согласно этой теореме при довольно общих условиях устанавливается асимптотическое равенство полных сечений рассеяния частишы и античастишы на одной и той же мишени. Работа [6] положила начало детальному изучению связи между поведением амплитуд рассеяния частицы и античастицы при высоких энергиях [7]-[26]. В развитие этого направления Логунов с сотрудниками внес выдающийся вклад. В его совместной с Нгуен Ван Хьеу и Хрусталевым работе было установлено, что асимптотическое равенство дифференциальных сечений рассеяния частицы и античастишы имеет

\footnotetext{
${ }^{*}$ Институт ядерных исследований РАН, Москва, Россия. E-mail: vernov@ms2.inr.ac.ru

${ }^{\dagger}$ Научно-исследовательский институт ядерной физики Московского государственного университета, Москва, Россия. E-mail: mnatsak@theory.sinp.msu.ru
} 
место при весьма общих условиях. Близкие результаты были получены в работах [12] и [13] (см. также работу [14]). В работе [17] был найден конечноэнергетический аналог этого результата, из которого, в частности, следует, что асимптотическое равенство дифференциальных сечений рассеяния частицы и античастицы для рассеяния вперед является строгим следствием только обших принципов теории.

Очень интересное следствие условий аналитичности и унитарности было найдено в работе [16], где было доказано, что асимптотически полные сечения рассеяния частицы и античастицы либо одновременно неограниченно возрастают (падают), либо оба ограничены сверху и снизу. В работах [20] было найдено, что когда эти сечения $\sigma_{ \pm}(s)$ неограниченно возрастают, то асимптотически $\sigma_{+}(s) / \sigma_{-}(s)=1$. Это соотношение было получено также в работах [15] и [22].

Обзор результатов, связанных с теоремой Померанчука и ее обобщениями, можно найти в [20], [27], [28].

Целью данной статьи является исследование при высоких энергиях свойств функции $\operatorname{Im} F_{+}(s, t) / \operatorname{Im} F_{-}(s, t)$, вытекающих из условий аналитичности, унитарности и кроссинг-симметрии, $F_{ \pm}(s, t)$ - амплитуды упругого рассеяния частицы (античастицы). В наиболее важном случае $t=0$ изучаемая функция сводится к $\sigma_{+}(s) / \sigma_{-}(s)$. В разделе 2 перечислены основные свойства функций $F_{ \pm}(s, t)$ и дан вывод соотношений $(8)$ и $(16)$, лежаших в основе полученных в работе результатов. В разделе 3 доказано, что в наиболее важном случае, когда полные сечения или не падают, или падают медленнее чем степенным образом, общее соотношение $\left|F_{+}(s, 0) / F_{-}(s, 0)\right| \rightarrow 1$ при $s \rightarrow \infty$ почти всегда сводится к $\sigma_{+}(s) / \sigma_{-}(s) \rightarrow 1$. Исключение составляет лишь физически довольно странный случай, когда $\left|\operatorname{Re} F_{ \pm}(s, 0) / \operatorname{Im} F_{ \pm}(s, 0)\right| \rightarrow \infty, s \rightarrow \infty$. Общее соотношение уточняется и в этом случае, а именно $\operatorname{Re} F_{+}(s, 0) / \operatorname{Re} F_{-}(s, 0) \rightarrow-1$. В действительности последнее соотношение выполняется, если только $\operatorname{Re} F_{ \pm}(s, 0) / \operatorname{Im} F_{ \pm}(s, 0) \nrightarrow 0$. В разделе 4 показано, как этот результат может быть усилен для непадающих или неограниченно растущих сечений. Доказано, что если $\sigma_{+}(s) \gg 4 \pi / t_{0}$, где $t_{0}-$ крайняя правая точка эллипса Мартена, то $\sigma_{+}(s) / \sigma_{-}(s) \cong 1$ (см. формулу (32)). Для большинства процессов рассеяния адронов $t_{0}=4 m_{\pi}^{2}$, т.е. указанное условие принимает вид $\sigma_{+}(s) \gg \pi / m_{\pi}^{2} \cong 2 \cdot 10^{-26} \mathrm{~cm}^{2}$. В случае неограниченно растущих сечений исследуется зависимость отношения $\sigma_{+}(s) / \sigma_{-}(s)$ от быстроты роста функций $\sigma_{ \pm}(s)$. В разделе 5 полученные результаты распространяются на случай конечных энергий. Это становится возможным благодаря выводу конечноэнергетического аналога неравенства Сингха и Роя [29]. В разделе 6 проанализированы различные предположения, приводящие к теоремам типа теоремы Померанчука. В частности, доказано, что если $\operatorname{Re} F_{ \pm}(s, 0)$ имеют один и тот же знак при $s \rightarrow \infty$, то разность полных сечений $\Delta \sigma(s)$ удовлетворяет ограничению $|\Delta \sigma(s)|<\pi^{2} /\left(2 m_{\pi}^{2}\right)$. Предлагаемая статья является развитием работы [30].

\section{2. ОСНОВНЫЕ ПРЕДПОЛОЖЕНИЯ И ОБЩИЕ РЕЗУЛЬТАТЫ}

Для упрощения формул, следуя Мартену, перейдем от переменной $s$ к практически совпадающей с ней при высоких энергиях переменной $\omega \equiv s+t / 2-m^{2}-M^{2}, m$ и $M-$ массы сталкиваюшихся частищ. 
Будем исходить из следующих, строго доказанных в аксиоматической теории поля, свойств амплитуд упругого рассеяния.

1. Функции $F_{ \pm}(\omega, t)$ аналитичны всюду в $\omega$-плоскости с разрезами $\left(-\infty,-\omega_{0}\right)$, $\left(\omega_{0}, \infty\right), \omega_{0}=2 m M+t / 2$. Отметим, что мы рассматриваем амплитуды рассеяния с вычтенными полюсными членами, которые пренебрежимо малы при высоких энергиях.

2.

$$
F_{ \pm}\left(\omega^{*}, t\right)=F_{ \pm}^{*}(\omega, t)
$$

3.

$$
F_{+}(-\omega, t)=F_{-}(\omega, t)
$$

Из формул (1) и (2) следует, что

$$
F_{+}\left(-\omega^{*}, t\right)=F_{-}^{*}(\omega, t)
$$

4.

$$
\operatorname{Im} F_{ \pm}(\omega, t) \geqslant 0, \quad \omega>\omega_{0} .
$$

Это условие заведомо выполнено, если $0 \leqslant t \leqslant t_{0}$, где $t_{0}-$ крайняя правая точка эллипса Мартена. При $t=0$ условие 4 является следствием оптической теоремы

$$
\operatorname{Im} F_{ \pm}(\omega, 0)=\frac{\omega}{8 \pi} \sigma_{ \pm}(\omega)
$$

где $\sigma_{ \pm}(\omega)$ - полные сечения рассеяния частицы и античастицы (в (5) опушены члены, несушественные при $\left.\omega \gg \omega_{0}\right)$.

5.

$$
\frac{F_{ \pm}(\omega, t)}{\omega^{2}} \rightarrow 0, \quad t<t_{0}
$$

6. Справедливо неравенство Фруассара-Мартена

$$
\left|F_{ \pm}(\omega, t)\right|<\frac{\omega \ln ^{2} \omega}{2 t_{0}}, \quad t \leqslant 0
$$

Для некоторых процессов аналитичность $F_{ \pm}(\omega, t)$ нарушается внутри некоторого круга вокруг начала координат, что, однако, не влияет на результаты, справедливые при асимптотических энергиях

Из перечисленных свойств функций $F_{ \pm}(\omega, t)$ следует, что

$$
\left|\frac{F_{ \pm}\left(\omega e^{i \varphi}, t\right)}{F_{ \pm}(\omega, t)}\right| \rightarrow 1, \quad \omega \rightarrow \infty, \quad 0<\varphi \leqslant \pi
$$

Поскольку все пределы рассматриваются при условии $\omega \rightarrow \infty$, ниже мы, как правило, не будем явно его указывать.

Строго говоря, соотношение (8) не обязано выполняться при всех асимптотических энергиях. Оно заведомо выполнено на некоторой последовательности $\omega_{i}, \omega_{i} \rightarrow \infty$ при 
$i \rightarrow \infty$. Условие (8) является следствием интегрального соотношения (см. ниже формулу (15)), из которого следует, что оно выполняется "в среднем" при $\omega \rightarrow \infty$. Такая ситуация типична для строгих ограничений, и ниже мы не будем это спешиально оговаривать.

Для доказательства условия (8) прежде всего отметим, что у функций $F_{ \pm}(\omega, t)$ нет нулей, если $F_{ \pm}\left(\omega_{0}, t\right)<0$. Перейдя, если необходимо, от $F_{ \pm}(\omega, t)$ к функциям $F_{ \pm}(\omega, t)-$ $F_{ \pm}\left(\omega_{0}, t\right)-\varepsilon$, практически совпадаюшим с первоначальными при высоких энергиях, мы можем считать, что

$$
F_{ \pm}\left(\omega_{0}, t\right)<0 .
$$

Заметим, что из дисперсионных соотношений (ДС) следует формула

$$
\operatorname{Im} \frac{F_{ \pm}(\omega, t)}{\omega}=A \operatorname{Im} \omega, \quad \text { где } A>0 .
$$

Действительно, согласно (6) ДС для $F_{ \pm}(\omega, t)$ требуют только двух вычитаний,

$$
F_{+}(\omega, t)=F_{+}(0, t)+F_{+}^{\prime}(0, t) \omega+\frac{\omega^{2}}{\pi} \int_{\omega_{0}}^{\infty} \frac{\operatorname{Im} F_{+}\left(\omega^{\prime}, t\right) d \omega^{\prime}}{\omega^{\prime 2}\left(\omega^{\prime}-\omega\right)}+\int_{\omega_{0}}^{\infty} \frac{\operatorname{Im} F_{-}\left(\omega^{\prime}, t\right) d \omega^{\prime}}{\omega^{\prime 2}\left(\omega^{\prime}+\omega\right)} .
$$

Из (11) следует, что

$$
\begin{aligned}
A= & -\frac{F_{+}(0, t)}{|\omega|^{2}}+\frac{1}{\pi} \int_{\omega_{0}}^{\infty} \frac{\operatorname{Im} F_{+}\left(\omega^{\prime}, t\right) d \omega^{\prime}}{\omega^{\prime}\left[\left(\omega^{\prime}-\operatorname{Re} \omega\right)^{2}+(\operatorname{Im} \omega)^{2}\right]}+ \\
& +\frac{1}{\pi} \int_{\omega_{0}}^{\infty} \frac{\operatorname{Im} F_{-}\left(\omega^{\prime}, t\right) d \omega^{\prime}}{\omega^{\prime}\left[\left(\omega^{\prime}+\operatorname{Re} \omega\right)^{2}+(\operatorname{Im} \omega)^{2}\right]} .
\end{aligned}
$$

Из свойства (4) следует, что $A>0$, так как $F_{+}(0, t)<0$, если $F_{ \pm}\left(\omega_{0}, t\right)<0$. Для доказательства отсутствия нулей у функций $F_{ \pm}(\omega, t)$ в интервале $\left(-\omega_{0}, \omega_{0}\right)$ достаточно предположить существование этих нулей и записать рассматриваемые функции в виде

$$
F_{ \pm}(\omega, t)=(\omega \mp \alpha)(\omega \mp \beta) \Phi_{ \pm}(\omega, t),
$$

так как согласно $(9)$ у функций $F_{ \pm}(\omega, t)$ может быть только четное число нулей. Написав ДС для $\Phi_{ \pm}(\omega, t)$, легко убедиться в том, что вследствие (4) $\Phi_{ \pm}(\omega, t)>0$ в интервале $\left(-\omega_{0}, \omega_{0}\right)$, а следовательно, и $F_{ \pm}\left(\omega_{0}, t\right)>0$, что противоречит условию $(9)$.

Неравенства (4) означают, что $\delta_{ \pm}(\omega, t)$ - фазы функций $F_{ \pm}(\omega, t)$ - удовлетворяют неравенствам

$$
0 \leqslant \delta_{ \pm}(\omega, t) \leqslant \pi
$$

Следовательно, согласно (10) имеем

$$
\varphi \leqslant \delta_{ \pm}\left(\omega e^{i \varphi}, t\right) \leqslant \pi+\varphi
$$

Рассмотрим интеграл

$$
\int_{C} \frac{\ln F_{+}\left(\omega^{\prime}, t\right) d \omega^{\prime}}{\omega^{\prime}}
$$


где контур $C$ состоит из отрезков $\left(\omega_{1}, \omega_{2}\right),\left(\omega_{2} e^{i \varphi}, \omega_{1} e^{i \varphi}\right)$ и дуг $\left(\omega_{1} e^{i \varphi}, \omega_{1}\right),\left(\omega_{2}, \omega_{2} e^{i \varphi},\right)$, $\omega_{1,2} \geqslant \omega_{0}$. Воспользовавшись теоремой Коши и неравенствами (14), получим

$$
\left|\int_{\omega_{1}}^{\omega_{2}} \ln \right| \frac{F_{+}\left(\omega^{\prime} e^{i \varphi}, t\right)}{F_{+}\left(\omega^{\prime}, t\right)}\left|\frac{d \omega^{\prime}}{\omega^{\prime}}\right| \leqslant \pi \varphi .
$$

Условие (8) выполняется, поскольку в (15) мы можем положить $\omega_{2}=\infty$. Когда $\varphi=\pi$, имеем согласно (3)

$$
\left|\frac{F_{-}(\omega, t)}{F_{+}(\omega, t)}\right| \rightarrow 1, \quad \omega \rightarrow \infty .
$$

\section{3. СВОЙСТВА ФУНКЦИИ $\operatorname{Im} F_{+}(\omega, t) / \operatorname{Im} F_{-}(\omega, t)$ ПРИ ВЫСОКИХ ЭНЕРГИЯХ}

Рассмотрим условия, при которых из (16) следует соотношение

$$
\left|\frac{\operatorname{Im} F_{-}(\omega, t)}{\operatorname{Im} F_{+}(\omega, t)}\right| \rightarrow 1 .
$$

УТВЕРЖДЕНИЕ 1. Предположим, что

$$
\omega^{1-\varepsilon}<\left|F_{+}(\omega, t)\right|<\omega^{1+\varepsilon}
$$

при $\omega \rightarrow \infty$. Условие (17) будет выполнено, если существует константа $C_{1}$ такая, что

$$
\left|\frac{\operatorname{Im} F_{+}(\omega, t)}{\operatorname{Re} F_{+}(\omega, t)}\right|>C_{1} .
$$

Если же существует такая константа $C_{2}$, что

$$
\left|\frac{\operatorname{Re} F_{+}(\omega, t)}{\operatorname{Im} F_{+}(\omega, t)}\right|>C_{2},
$$

mo

$$
\left|\frac{\operatorname{Re} F_{-}(\omega, t)}{\operatorname{Re} F_{+}(\omega, t)}\right| \rightarrow-1 .
$$

Отметим, что в наиболее важном случае $t=0$ условие (18) сводится к условию

$$
\sigma_{+}(\omega)>\omega^{-\varepsilon} .
$$

Заметим также, что условие (19) эквивалентно более удобному для проверки условию

$$
\frac{\operatorname{Im} F_{+}(\omega, t)}{\left|F_{+}(\omega, t)\right|}>C_{1}^{\prime} .
$$

Константы $C_{i}$ могут быть произвольно малы. Поэтому если функции $\sigma_{ \pm}(\omega)$ не падают степенным образом, то $\sigma_{+}(\omega) / \sigma_{-}(\omega) \rightarrow 1$ за исключением физически маловероятного случая, когда в асимптотике полные сечения "вымирают" по сравнению с дифференциальными сечениями рассеяния вперед. 
ДокАЗАТЕЛЬСТво. Воспользуемся ДС для $\ln \left(F_{+}(\omega, t) F_{-}(\omega, t)\right)$. Легко проверить, что согласно (1) и (9)

$$
\delta_{+}\left(\omega^{*}, t\right)=-\delta_{-}\left(\omega^{*}, t\right)+2 \pi .
$$

Поэтому

$$
\ln \frac{F_{+}(\omega, t) F_{-}(\omega, t)}{F_{+}(0, t) F_{-}(0, t)}=\frac{2 \omega^{2}}{\pi} \int_{\omega_{0}}^{\infty} \frac{\tilde{\delta}\left(\omega^{\prime}, t\right) d \omega^{\prime}}{\omega^{\prime}\left(\omega^{\prime 2}-\omega^{2}\right)}
$$

где $\tilde{\delta}(\omega, t)=\delta_{+}(\omega, t)+\delta_{-}(\omega, t)-2 \pi$. Удобно записать $\delta_{ \pm}(\omega, t)$ в виде

$$
\delta_{ \pm}(\omega, t)=\frac{\pi}{2}+\gamma_{ \pm}(\omega, t)
$$

Согласно (13) имеем

$$
\left|\gamma_{ \pm}(\omega, t)\right| \leqslant \frac{\pi}{2}
$$

Рассматривая равенство (24) в точке $i \omega, \omega>0$, запишем его в виде

$$
\ln \left[\frac{F_{+}(i \omega, t) F_{-}(i \omega, t)}{F_{+}(0, t) F_{-}(0, t)} \frac{\omega_{0}^{2}}{\omega_{0}^{2}+\omega^{2}}\right]=\frac{2}{\pi} \int_{\omega_{0}}^{\omega} \frac{\gamma_{+}\left(\omega^{\prime}, t\right)+\gamma_{-}\left(\omega^{\prime}, t\right)}{\omega^{\prime}} d \omega^{\prime}+D(\omega) .
$$

Из $\left(13^{\prime}\right)$ следует оценка

$$
|D(\omega)|<2 \ln 2 .
$$

Согласно (25) и (8) условие (18) выполнено, если только

$$
\gamma_{+}\left(\omega^{\prime}, t\right)+\gamma_{-}\left(\omega^{\prime}, t\right) \rightarrow 0
$$

Следовательно, или $\gamma_{ \pm}(\omega, t) \rightarrow 0$, или

$$
\frac{\gamma_{+}(\omega, t)}{\gamma_{-}(\omega, t)} \rightarrow-1
$$

Таким образом,

$$
\frac{\operatorname{Re} F_{+}(\omega, t)}{\operatorname{Im} F_{+}(\omega, t)} \rightarrow-\frac{\operatorname{Re} F_{-}(\omega, t)}{\operatorname{Im} F_{-}(\omega, t)},
$$

если выполнено условие (19). Мы видим, что когда неравенства (18) и (20) выполнены одновременно, то справедливо как соотношение (17), так и соотношение (21). Утверждение доказано. 
ЗАмЕчАниЕ 1. Если неравенства (18) и неравенство (22) заменить более сильными неравенствами

$$
\begin{gathered}
\omega(\ln \omega)^{-m}<\left|F_{ \pm}(\omega, t)\right|<\omega(\ln \omega)^{m} \\
\sigma_{+}(\omega)>(\ln \omega)^{-m}
\end{gathered}
$$

то условие (19) может быть заменено более слабым условием

$$
\left|\frac{\operatorname{Im} F_{+}(\omega, t)}{\operatorname{Re} F_{+}(\omega, t)}\right| \ln \omega \rightarrow \infty .
$$

Действительно, в этом случае

$$
\left|\gamma_{+}(\omega, t)+\gamma_{-}(\omega, t)\right| \leqslant o\left(\frac{1}{\ln \omega}\right) .
$$

С другой стороны, согласно (28) имеем

$$
\gamma_{+}(\omega, t)= \pm \frac{\pi}{2}+O_{+}\left(\frac{1}{\ln \omega}\right)
$$

Тогда

$$
\gamma_{-}(\omega, t)=\mp \frac{\pi}{2}+O_{-}\left(\frac{1}{\ln \omega}\right)
$$

и, следовательно,

$$
O_{+}\left(\frac{1}{\ln \omega}\right) / O_{-}\left(\frac{1}{\ln \omega}\right) \rightarrow-1
$$

Оказывается, что вследствие аналитичности в $t$-плоскости и унитарности условие (28) выполняется всегда, когда $\sigma_{+}(\omega) \rightarrow \infty$. Напомним, что согласно результату, полученному в [20], в этом случае и $\sigma_{-}(\omega) \rightarrow \infty$. Действительно, в работе [29] было получено неравенство

$$
\left|F_{ \pm}(\omega, 0)\right| \leqslant \frac{\omega}{4 \sqrt{\pi t_{0}}} \sqrt{\sigma_{ \pm}(\omega)} \ln \frac{\omega}{\sigma_{ \pm}(\omega)}
$$

из которого непосредственно следует $\left(18^{\prime}\right)$, если $\sigma_{ \pm}(\omega) \rightarrow \infty$.

В следуюшем разделе мы детально рассмотрим поведение функции $\sigma_{+}(\omega) / \sigma_{-}(\omega)$ в случае непадаюших полных сечений.

В заключение этого раздела кратко рассмотрим случай, когда функции $F_{ \pm}(\omega, t) / \omega$ растут или падают степенным образом. Если $\operatorname{Im} F_{+}(\omega, t) / \operatorname{Im} F_{-}(\omega, t) \rightarrow 1$, то из соотношения (16) следует, что также и $\operatorname{Re} F_{+}(\omega, t) / \operatorname{Re} F_{-}(\omega, t) \rightarrow 1$. Действительно, случаи $\operatorname{Re} F_{ \pm}(\omega, t) / \operatorname{Im} F_{ \pm}(\omega, t) \rightarrow 0$ и $\operatorname{Re} F_{+}(\omega, t) / \operatorname{Re} F_{-}(\omega, t) \rightarrow-1$ исключены, поскольку в первом случае $\operatorname{Re} F_{s}(\omega, t) / \operatorname{Im} F_{s}(\omega, t) \rightarrow 0$, а во втором - $\left|\operatorname{Re} F_{a}(\omega, t) / \operatorname{Im} F_{a}(\omega, t)\right| \rightarrow \infty$, где $F_{s}(\omega, t) \equiv F_{+}(\omega, t)+F_{-}(\omega, t), F_{a}(\omega, t) \equiv F_{+}(\omega, t)-F_{-}(\omega, t)$. Чтобы убедиться в том, что вследствие перекрестной симметрии последние условия не могут быть выполнены, достаточно рассмотреть случай чисто степенных асимптотик функций $F_{s}(\omega, t) / \omega$ и $F_{a}(\omega, t) / \omega$, удовлетворяюших условию перекрестной симметрии. 


\section{4. СВЯЗЬ МЕЖДУ ПОВЕДЕНИЕМ НЕПАДАЮШИХ ПОЛНЫХ СЕЧЕНИЙ ЧАСТИЦЫ И АНТИЧАСТИЦЫ}

Покажем, что асимптотически $\sigma_{+}(\omega) / \sigma_{-}(\omega) \cong 1$, когда $\sigma_{+}(\omega) \gg 4 \pi / t_{0}$.

УТВЕРЖДЕнИЕ 2. Пусть при $\omega>\omega_{1}$, где $\omega_{1}$ - некоторая әнергия,

$$
\sigma_{+}(\omega) \geqslant C_{+} \frac{4 \pi}{t_{0}}
$$

тогда

$$
\sigma_{-}(\omega) \geqslant C_{-} \frac{4 \pi}{t_{0}}
$$

әде $C_{-}$определено неравенством

$$
\frac{C_{+}}{C_{-}}-1 \leqslant \frac{\pi}{\sqrt{C_{-}}}
$$

Точный смысл неравенства (31) состоит в том, что противоположное неравенство

$$
\sigma_{-}(\omega) \leqslant C_{-} \frac{4 \pi}{t_{0}}
$$

может быть справедливо только, если $C_{-}$удовлетворяет условию (32).

ДОкАЗАТЕЛЬСТво. Рассмотрим ДС для $F_{a}(\omega, 0)$ в чисто мнимой точке $i \omega$,

$$
F_{a}(i \omega, 0)=-2 i \operatorname{Im} F_{-}(i \omega, 0)=i \omega F_{a}^{\prime}(0,0)-\frac{2 i \omega^{3}}{\pi} \int_{\omega_{0}}^{\infty} \frac{\operatorname{Im} F_{a}\left(\omega^{\prime}, 0\right) d \omega^{\prime}}{\omega^{\prime 2}\left(\omega^{\prime 2}+\omega^{2}\right)}
$$

Первое равенство в (34) следует из (1) и (2). Предположим справедливость неравенства (33) при $\omega>\omega_{1}$. Оценка интеграла в (34) приводит к неравенству

$$
\operatorname{Im} F_{-}(i \omega, 0)>\frac{1}{2 \pi t_{0}}\left(C_{+}-C_{-}\right) \omega \ln \frac{\omega}{\omega_{1}}(1+\varepsilon(\omega)),
$$

где поправка $\varepsilon(\omega) \rightarrow 0$ при $\omega \rightarrow \infty$ (ниже мы эту поправку не выписываем). Согласно соотношению (8) неравенство (35) означает, что

$$
\left|F_{-}(\omega, 0)\right|>\frac{1}{2 \pi t_{0}}\left(C_{+}-C_{-}\right) \omega \ln \frac{\omega}{\omega_{1}}
$$

Сравнивая (36) и (33), приходим к требуемому неравенству (32). Утверждение доказано. 
ЗАмечаниЕ 2. Соотношение (32) является оптимальным. Действительно, заменяя знаки неравенств в $(30)$ и (33) равенствами, получим из ДС для $F_{s}(\omega, 0)$, что $F_{s}(i \omega, 0) \sim$ $\left(C_{+}+C_{-}\right) \omega$. Следовательно,

$$
\left|F_{-}(i \omega, 0)\right|=2\left|\operatorname{Im} F_{a}(i \omega, 0)\right|\left(1+\varepsilon_{1}(\omega)\right), \quad \varepsilon_{1}(\omega) \rightarrow 0
$$

т.е. неравенство (36) не может быть усилено. Неравенство (29) также оптимально (см. ниже раздел 5$)$.

Получим теперь дополнительное ограничение на $\sigma_{+}(\omega) / \sigma_{-}(\omega)$ в случае неограниченно растущих полных сечений.

УТВЕРЖДЕНИЕ 3. Если при высоких энергиях $\left(\omega>\omega_{1}\right)$ выполняется неравенствo

$$
\sigma_{+}(\omega)>\frac{4 \pi}{t_{0}} C_{+}(\ln \omega)^{\alpha}, \quad \alpha>0
$$

mo

$$
\sigma_{-}(\omega)>\frac{4 \pi}{t_{0}} C_{+}(\ln \omega)^{\alpha}-\frac{2 \pi^{2}}{t_{0}}(2+\alpha) \sqrt{C_{+}}(\ln \omega)^{\alpha / 2}
$$

Для определенности считаем, что $\sigma_{+}(\omega)>\sigma_{-}(\omega)$ при $\omega>\omega_{1}$.

ДокАЗАТЕЛЬСТво. Заметим, что если справедливо неравенство, противоположное (38), то

$$
\Delta \sigma(\omega)>\frac{4 \pi}{t_{0}} C(\ln \omega)^{\beta}, \quad \beta \geqslant \frac{\alpha}{2}, \quad C \geqslant(2+\alpha) \pi \sqrt{C_{+}} .
$$

Однако из формул (34) и (8) следует, что

$$
\left|F_{-}(\omega, 0)\right|>\frac{1}{2 \pi t_{0}} C \omega \frac{(\ln \omega)^{\beta+1}}{\beta+1}(1+\varepsilon(\omega)) .
$$

С другой стороны, согласно неравенству (29) имеем

$$
\left|F_{-}(\omega, 0)\right|<\frac{1}{4 \sqrt{\pi} t_{0}} \sqrt{C_{+}}(\ln \omega)^{\alpha / 2+1}
$$

Сравнивая формулы (40) и (41), видим, что утверждение доказано.

Заметим, что из полученного ограничения $(38)$ следует, что когда $\sigma_{-}(\omega) \rightarrow\left(4 \pi / t_{0}\right) \times$ $C_{+}(\ln \omega)^{\alpha}$, то

$$
\frac{\sigma_{-}(\omega)}{\sigma_{+}(\omega)}>1-\frac{\pi(2+\alpha)}{2 \sqrt{C_{+}}}(\ln \omega)^{-\alpha / 2}
$$




\section{5. ПОВЕДЕНИЕ ФУНКЦИИ $\sigma_{+}(\omega) / \sigma_{-}(\omega)$ ПРИ ВЫСОКИХ, НО КОНЕЧНЫХ ЭНЕРГИЯХ}

Покажем теперь, что для ряда процессов полученные выше асимптотические результаты могут быть перенесены на конечные энергии. Заметим, что неравенство (15) является конечноэнергетическим и, следовательно, существует конечноэнергетический аналог соотношения (8). В свою очередь, и неравенство (35) является конечноэнергетическим, поскольку $\varepsilon(\omega) \ll 1$ при достаточно больших, но конечных энергиях. Единственное асимптотическое соотношение, которое было использовано - неравенство (29). Покажем, что для этого неравенства существует конечноэнергетический аналог.

Исходным пунктом для вывода неравенства (29) является условие (6). Поэтому прежде всего надо найти конечноэнергетический аналог этого условия. Для этого заметим, что согласно (6)

$$
\int_{\omega_{0}}^{\infty} \frac{\operatorname{Im} F_{+}(\omega, t)}{\omega^{3}} d \omega \equiv A<\infty, \text { если } 0<t<t_{0} .
$$

Воспользуемся разложением функции $\operatorname{Im} F_{+}(\omega, t)$ в ряд по полиномам Лежандра,

$$
\operatorname{Im} F_{+}(\omega, t)=2 \sum_{l=0}^{\infty}(2 l+1) \operatorname{Im} f_{l}(\omega) P_{l}(x), \quad x=1+\frac{2 t}{\omega},
$$

в формуле (43) опушены множители, несушественные при $\omega \gg \omega_{0}$. Условие унитарности дает

$$
\operatorname{Im} f_{l}(\omega) \geqslant\left|f_{l}(\omega)\right|^{2}
$$

Известно также, что $P_{l}(x)>0$, если $x>1$, т.е. $\operatorname{Im} F_{+}(\omega, t)>0$.

Из $(42)$ следует, что для любого интервала $(\omega, \nu \omega)$ справедливо неравенство

$$
\operatorname{Im} F_{+}(\bar{\omega}, t)<\frac{\bar{\omega}^{2} A}{\ln \nu} \equiv \bar{\omega}^{2} d, \quad \bar{\omega} \in(\omega, \nu \omega)
$$

Ниже мы опускаем черту у $\bar{\omega}$. Для ряда процессов постоянную $A$ в неравенстве (45) можно оценить. Для $(\pi-\pi)$-рассеяния сушествуют строгие ограничения на $A[28]$. Для $(\pi-N)$-рассеяния $A$ определяется величиной $d$-волны в $t$-канале [31]. Отметим, что поскольку $\sigma_{+}(\omega)$ - медленно меняющаяся функция (по крайней мере, при современных энергиях), то выбранный интервал мал с физической точки зрения, если $\nu-1 \ll 1$.

Неравенство (29) ограничивает максимум модуля $\left|F_{+}(\omega, 0)\right|$ при следующих дополнительных условиях: выполнено соотношение $(6)$ и функция $\operatorname{Im} F_{+}(\omega, 0)$ предполагается заданной. При конечных энергиях вместо (6) используется неравенство (45). Поскольку искомый максимум тем больше, чем больше $\operatorname{Im} F_{+}(\omega, t)$, то неравенство $(45)$ следует заменить равенством. Этот максимум реализуется при некотором поведении $f_{l}(\omega)$, которое определяется с помощью метода неопределенных множителей Лагранжа (см. [32] и [29]). Из условия унитарности (44) следует, что $\left|F_{+}(\omega, 0)\right| \leqslant \Phi(\omega, 0)$, где

$$
\Phi(\omega, 0)=2 \sum_{l=0}^{\infty}(2 l+1) b_{l}(\omega), \quad b_{l}(\omega)=\sqrt{\operatorname{Im} f_{l}(\omega)} .
$$


Построим функцию

$$
L=\Phi(\omega, 0)+\alpha \operatorname{Im} F_{+}(\omega, t)+\beta \operatorname{Im} F_{+}(\omega, 0),
$$

где $\alpha$ и $\beta$ - неопределенные множители Лагранжа. Искомый режим $b_{l}(\omega)$ найдем из условия $\partial L / \partial b_{l}(\omega)=0$ (этот режим соответствует именно $\max \Phi(\omega, 0)$, что доказывается с помошью рассуждений, аналогичных проведенным в работе [29]). В результате получим

$$
b_{l}(\omega)=\frac{a}{b+P_{l}(x)}, \quad a=-\frac{1}{2 \alpha}, \quad b=\frac{\beta}{\alpha} .
$$

Осталось вычислить константы $a$ и $b$. Мы сделаем это в наиболее важном случае, когда $\sigma_{+}(\omega) \gg 4 \pi / t_{0}$. Тогда основной вклад в парциальные амплитуды дают значения $l$, удовлетворяющие условию

$$
2 l \sqrt{\frac{t}{\omega}} \equiv \gamma \gg 1
$$

При таких $\gamma$ (фактически, начиная с $\gamma=1$ ) функции $P_{l}(x)$ с хорошей точностью аппроксимируются асимптотической формулой ([31], формула (15))

$$
P_{l}(x)=\frac{e^{\gamma}}{\sqrt{2 \pi \gamma}} .
$$

Заменяя суммирование в формулах (43) и (46) интегрированием и учитьвая, что фактически $\gamma \geqslant 1$, приходим согласно (48) к следуюшим выражениям:

$$
\begin{aligned}
\operatorname{Im} F_{+}(\omega, t) & =a^{2} \sqrt{2 \pi} \frac{\omega}{t} \int_{1}^{\infty} \frac{\gamma^{3 / 2} e^{\gamma} d \gamma}{\left(b \sqrt{2 \pi \gamma}+e^{\gamma}\right)^{2}} \\
\operatorname{Im} F_{+}(\omega, 0) & =a^{2} 2 \pi \frac{\omega}{t} \int_{1}^{\infty} \frac{\gamma^{2} d \gamma}{\left(b \sqrt{2 \pi \gamma}+e^{\gamma}\right)^{2}}, \\
\Phi(\omega, 0) & =a^{2} \sqrt{2 \pi} \frac{\omega}{t} \int_{1}^{\infty} \frac{\gamma^{3 / 2} d \gamma}{b \sqrt{2 \pi \gamma}+e^{\gamma}} .
\end{aligned}
$$

Условие $\sigma_{\text {tot }}(\omega) \gg 4 \pi / t_{0}$ означает, что $\operatorname{Im} F_{+}\left(\omega, t_{0}\right) / \operatorname{Im} F_{+}(\omega, 0) \gg 1$ (см. [28], формулы (6.8), (6.9) и (6.12)). В асимптотике это условие тривиально выполняется, достаточно сравнить соотношения (45) и $(7)$. При таком поведении $\sigma_{+}(\omega)$ оказывается, что $b \gg 1$, что позволяет легко оценить интегралы в формулах (50)-(52). Можно показать, что с точностью до поправочных членов, которые становятся пренебрежимо малыми при $\omega \rightarrow \infty$, имеют место соотношения

$$
\begin{aligned}
\operatorname{Im} F_{+}(\omega, t) & =a^{2} \frac{\omega}{t} \frac{\ln b}{b}, \\
\operatorname{Im} F_{+}(\omega, 0) & =a^{2} \frac{\omega}{2 t} \frac{\ln ^{2} b}{b^{2}}, \\
\Phi(\omega, 0) & =a \frac{\omega}{2 t} \frac{\ln ^{2} b}{b} .
\end{aligned}
$$

Из (53)-(55) легко найти конечноэнергетический аналог неравенства (29),

$$
\left|F_{+}(\omega, 0)\right| \leqslant \frac{\omega}{4 \sqrt{\pi t}} \sqrt{\sigma_{+}(\omega)} \ln \frac{4 \pi d \omega}{\sigma_{+}(\omega)} .
$$

$\mathrm{B}$ асимптотике можно положить $t=t_{0}$. При конечных энергиях выбор $t$ зависит от рассматриваемых процессов и интервалов энергий. 


\section{6. РАЗЛИЧНЫЕ ВАРИАНТЫ ТЕОРЕМ ТИПА ТЕОРЕМЫ ПОМЕРАНЧУКА}

В этом разделе мы проанализируем соотношение $\Delta \sigma(\omega) \rightarrow 0$ и вьведем новые ограничения на $\Delta \sigma(\omega)$. Заметим, что $\Delta \sigma(\omega) \rightarrow 0$ тогда и только тогда, когда $F_{a}(\omega, 0) /(\omega \ln \omega) \rightarrow 0$. Действительно, если последнее условие выполнено, то согласно теореме Фрагмена-Линделёфа (см., например, [10], [14]) имеет место условие $F_{a}(i \omega, 0) /(\omega \ln \omega) \rightarrow 0$. Согласно (35) это условие не выполняется, когда $\Delta \sigma(\omega)>$ $\Delta>0$. Наоборот, если $\Delta \sigma(\omega)>\Delta$, то $F_{a}(i \omega, 0) /(\omega \ln \omega) \nrightarrow 0$, откуда следует, что и $F_{a}(\omega, 0) /(\omega \ln \omega) \nrightarrow 0$. Условие $F_{a}(\omega, 0) /(\omega \ln \omega) \rightarrow 0$ можно заменить на условие $\operatorname{Re} F_{a}(\omega, 0) /(\omega \ln \omega) \rightarrow 0$ (см. [28], § 3 или [27]). Последнее условие выполняется очевидным образом, если $\operatorname{Re} F_{ \pm}(\omega, 0) /(\omega \ln \omega) \rightarrow 0$. Если же предполагать, что только $\operatorname{Re} F_{+}(\omega, 0) /(\omega \ln \omega) \rightarrow 0$, то сушествует возможность того, что $\operatorname{Re} F_{-}(\omega, 0) \sim \omega \ln \omega$, однако

$$
\operatorname{Re} F_{-}(\omega, 0) \leqslant \frac{\pi}{2 t_{0}} \omega \ln \omega
$$

Действительно, аналогичное ограничение выполнено для $\operatorname{Re} F_{s}(\omega, 0) / 2$ (см., например, $[28], \S 3)$. Поскольку в рассматриваемом случае $\operatorname{Im} F_{a}(\omega, 0) \sim \operatorname{Re} F_{a}(\omega, 0) / \ln \omega$, то условие (57) можно заменить на условие

$$
\left|F_{a}(i \omega, 0)\right| \leqslant \frac{\pi}{2 t_{0}} \omega \ln \omega
$$

Тогда согласно (35) имеем

$$
|\Delta \sigma(\omega)| \leqslant \frac{2 \pi^{3}}{t_{0}}
$$

При этом $\sigma_{ \pm}(\omega) \sim(\ln \omega)^{2}$, поскольку если $\operatorname{Re} F_{s}(\omega, 0) \sim \omega \ln \omega$, то $\operatorname{Im} F_{s}(\omega, 0) \sim \omega \ln ^{2} \omega$ $([28], \S 3)$. Неравенство (58) выполняется всегда, когда $\operatorname{Re} F_{ \pm}(\omega, 0)$ имеют один и тот же знак, поскольку тогда справедливы неравенства $(57)$ и $\left(57^{\prime}\right)$.

В заключение отметим, что все ограничения на функцию $\Delta \sigma(\omega)$ являются следствием условия перекрестной симметрии и представляют собой связь между верхними или нижними границами для функций $\operatorname{Im} F_{a}(\omega, 0)$ и $\operatorname{Re} F_{a}(\omega, 0)$. Аналитичность в $t$-плоскости и унитарность используются лишь неявно (предполагается выполненным неравенство Фруассара-Мартена).

Условие $\Delta \sigma(\omega) \rightarrow 0$ нетривиально лишь для таких амплитуд, для которых $F_{a}(\omega, 0) /$ $(\omega \ln \omega) \rightarrow 0, \sigma_{ \pm}(\omega) \nrightarrow 0$, т.е. для сравнительно узкого класса амплитуд. Условие $\sigma_{+}(\omega) /$ $\sigma_{-}(\omega) \rightarrow 1$ носит сушественно более обший характер, оно справедливо всегда, когда $\sigma_{ \pm}(\omega) \rightarrow \infty$. При $\sigma_{ \pm}(\omega) \gg 4 \pi / t_{0}$ это условие вьполняется с хорошей точностью. Во всех случаях, когда допустимо только медленное падение сечений (22), оно справедливо, если выполнено условие (19).

2 Теоретическая и математическая физика, т. 132, № 2, 2002 г. 


\section{Список литературы}

[1] Н. Н. Боголюбов, Б. В. Медведев, М. К. Поливанов. Вопросы теории дисперсионных соотношений. М.: Физматгиз, 1958.

[2] H. J. Bremermann, R. Oehme, J. G. Taylor. Phys. Rev. 1958. V. 109. P. 2178.

[3] R. Oehme. Mod. Phys. Lett. 1993. V. 13. P. 1533; $\pi N$-Newsletter. 1992. V. 7. P. 1.

[4] H. Lehmann. Nuovo Cimento. 1958. V. 10. P. 579.

[5] A. Martin. Nuovo Cimento. 1966. V. 42. P. 930.

[6] И. Я. Померанчук. ЖЭТФ. 1958. Т. 34. С. 725.

[7] D. Amati, M. Fierz, V. Glaser. Phys. Rev. Lett. 1960. V. 4. P. 89.

[8] M. Sugawara, A. Kanazawa. Phys. Rev. 1961. V. 123. P. 1995.

[9] S. Weinberg. Phys. Rev. 1961. V. 124. P. 2049.

[10] H. Н. Мейман. ЖЭЭФ. 1962. Т. 43. С. 2277; 1975. Т. 68. С. 791.

[11] A. A. Logunov, Nguyen Van Hieu, I. T. Todorov, O. A. Khrustalev. Phys. Lett. 1963. V. 7. Р. $69 ;$ Р. 252; А. А. Логунов, Нгуен Ван Хьеу, И. Т. Тодоров, О. А. Хрусталев. ЖЭТФ. 1964. Т. 46. С. 1079; А. А. Логунов, Нгуен Ван Хьеу, И. Т. Тодоров. УФН. 1966. T. 88. C. 51.

[12] L. van Hove. Phys. Lett. 1964. V. 3. P. 63.

[13] Н. Н. Мейман. ЖЭЭТ. 1964. Т. 46. С. 1039.

[14] A. Martin. Nuovo Cimento. 1965. V. 39. P. 704.

[15] R. J. Eden. Phys. Rev. Lett. 1966. V. 16. P. 39.

[16] Г. Г. Волков, А. А. Логунов, М. А. Мествиришвили. ТМФ. 1970. Т. 4. С. 196.

[17] Ю. С. Вернов. ТМФ. 1970. Т. 4. С. 3.

[18] S. M. Roy, V. Singh. Phys. Lett. B. 1970. V. 32. P. 50.

[19] T. Kinoshita. Phys. Rev. D. 1970. V. 2. P. 2346.

[20] А. А. Логунов, М. А. Мествиришвили, О. А. Хрусталев. ТМФ. 1971. Т. 9. С. 153; ЭЧАЯ. 1972. Т. 3. С. 3; С. 515.

[21] H. Cornille, A. Martin. Phys. Lett. B. 1972. V. 40. P. 671; Nucl. Phys. B. 1972. V. 49. P. 413.

[22] Tran N. Truong, W. S. Lam. Phys. Rev. D. 1972. V. 6. P. 2875; G. Grunberg, Tran N. Truong. Phys. Rev. D. 1974. V. 9. P. 2874; Phys. Rev. Lett. 1973. V. 31. P. 63.

[23] R. Wit. Acta Phys. Slov. 1973. V. 23. P. 203.

[24] J. Fischer et al. Phys. Rev. D. 1976. V. 13. P. 133; J. Fischer, P. Kolár̆. Phys. Rev. D. 1978. V. 17. P. 2168

[25] Ю. С. Вернов, М. Н. Мнацаканова. ТМФ. 1978. Т. 34. С. 153.

[26] Ю. М. Ломсадзе, Б. А. Аграновский. ЯФ. 1979. Т. 30. С. 754.

[27] J. Fischer J., R. Šály. Fortschr. Phys. 1980. V. 28. P. 237; J. Fischer. Phys. Rep. 1981. V. 76. P. 157.

[28] Ю. С. Вернов, М. Н. Мнацаканова. ЭЧАЯ. 2001. Т. 32. С. 1115.

[29] V. Singh, S. M. Roy. Ann. Phys. 1970. V. 57. P. 461.

[30] Yu. S. Vernov, M. N. Mnatsakanova. Relations between the elastic scattering amplitude of the particle and the antiparticle at high energies. In: Proc. of the XIII Workshop "Problems on high energy physics and field theory", Protvino, 1990. Ed. S. M. Troshin. M.: Nauka, 1991. P. 205.

[31] Ю. С. Вернов, М. Н. Мнацаканова. ТМФ. 1982. Т. 52. С. 199.

[32] M. Einhorn, R. Blankenbecler. Ann. Phys. 1971. V. 67. P. 480.

Поступила в редакцию 14.III.2002 г. 